\title{
An artificial neural network for automated behavioral state classification in rats
}

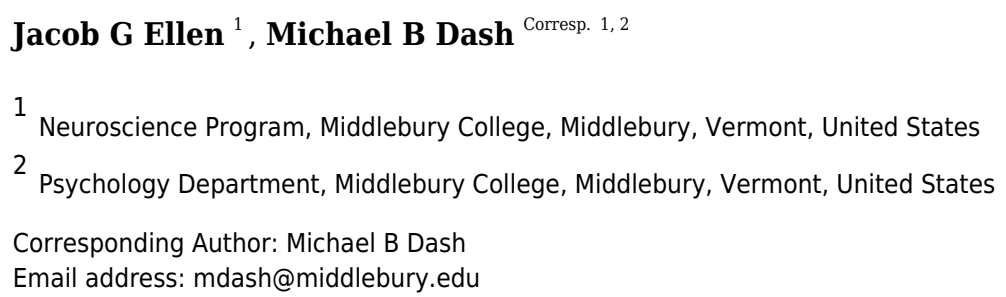

Accurate behavioral state classification is critical for many research applications. Researchers typically rely upon manual identification of behavioral state through visual inspection of electrophysiological signals, but this approach is time intensive and subject to low inter-rater reliability. To overcome these limitations, a diverse set of algorithmic approaches have been put forth to automate the classification process. Recently, novel machine learning approaches have been detailed that produce rapid and highly accurate classifications. These approaches however, are often computationally expensive, require significant expertise to implement, and/or require proprietary software that limits broader adoption. Here we detail a novel artificial neural network that uses electrophysiological features to automatically classify behavioral state in rats with high accuracy, sensitivity, and specificity. Common parameters of interest to sleep scientists, including statedependent power spectra and homeostatic non-REM slow wave activity, did not significantly differ when using this automated classifier as compared to manual scoring. Flexible options enable researchers to further increase classification accuracy through manual rescoring of a small subset of time intervals with low model prediction certainty or further decrease researcher time by generalizing trained networks across multiple recording days. The algorithm is fully open-source and coded within a popular, and freely available, software platform to increase access to this research tool and provide additional flexibility for future researchers. In sum, we have developed a readily implementable, efficient, and effective approach for automated behavioral state classification in rats. 


\section{An Artificial Neural Network for Automated Behavioral State Classification in Rats}

2 Jacob Gould Ellen ${ }^{1}$ and Michael Bennett Dash ${ }^{1,2}$

$3 \quad{ }^{1}$ Neuroscience Program, Middlebury College, Middlebury, VT, USA

4 2Department of Psychology, Middlebury College, Middlebury, VT, USA

6 Corresponding author:

7 Dr. Michael Dash

8276 Bicentennial Way

9281 McCardell Bicentennial Hall

10 Middlebury, VT 05753, USA

11 mdash@middlebury.edu

\section{Abstract}

Accurate behavioral state classification is critical for many research applications. Researchers typically rely upon manual identification of behavioral state through visual inspection of electrophysiological signals, but this approach is time intensive and subject to low inter-rater reliability. To overcome these limitations, a diverse set of algorithmic approaches have been put forth to automate the classification process. Recently, novel machine learning approaches have been detailed that produce rapid and highly accurate classifications. These approaches however, are often computationally expensive, require significant expertise to implement, and/or require proprietary software that limits broader adoption. Here we detail a novel artificial neural network that uses electrophysiological features to automatically classify behavioral state in rats with high accuracy, sensitivity, and specificity. Common parameters of interest to sleep scientists, including state-dependent power spectra and homeostatic non-REM slow wave activity, did not significantly differ when using this automated classifier as compared to manual scoring. Flexible options enable researchers to further increase classification accuracy through manual rescoring of a small subset of time intervals with low model prediction certainty or further decrease researcher time by generalizing trained networks across multiple recording days. The algorithm is fully open-source and coded within a popular, and freely available, software platform to increase access to this research tool and provide additional flexibility for future researchers. In sum, we have developed a readily implementable, efficient, and effective approach for automated behavioral state classification in rats. 


\section{Introduction}

State-dependent differences in neuronal activity contribute to widespread changes in information processing and conscious awareness and ultimately appear to subserve many important functions for maintaining brain health (Hauglund, Pavan, \& Nedergaard, 2020; Nir, Massimini, Boly, \& Tononi, 2013; Quilichini \& Bernard, 2012). Characterizing and identifying these state-dependent activity patterns is therefore a critical task for addressing a diverse set of research questions. Manual classification of behavioral state using visual scoring of the electroencephalogram (EEG), local field potential (LFP), and/or electromyogram (EMG) is often performed, yet has significant limitations as compared to algorithmic classifiers. Specifically, manual classification is time intensive and scales proportionally with the size of a data set, ultimately relies upon subjective determinations that limit inter-rater reliability, and requires extensively trained researchers to achieve high classification accuracies (Gao, Turek, \& Vitaterna, 2016; Gross et al., 2009; Miladinović et al., 2019; Rytkönen, Zitting, \& Porkka-Heiskanen, 2011; Stephenson, Caron, Cassel, \& Kostela, 2009).

Many promising automatic behavioral state classifiers have been developed in recent years. Although each of these classifiers typically relies upon characteristics derived from similar electrophysiological signals (i.e. EEG, LFP, and EMG), they differ as a function of how those characteristics are selected and combined to predict behavioral state. Semi-automated approaches, including user defined logic rules/thresholds (Gross et al., 2009) and principal component analyses (Gilmour, Fang, Guan, \& Subramanian, 2010), accurately predict behavioral state using researcher-identified electrophysiological features. Supervised learning algorithms similarly classify behavioral state based on handcrafted features and expert classifications, but are able to automatically "learn" rules for combining those features to accurately predict behavioral state. Successful supervised classifiers have been implemented through support vector machines (Crisler, Morrissey, Anch, \& Barnett, 2008), naïve Bayes classifiers (Rempe, Clegern, \& Wisor, 2015; Rytkönen et al., 2011), decision trees and linear discriminant analysis (Brankack, Kukushka, Vyssotski, \& Draguhn, 2010) and an ensemble method that uses all of the above (Gao et al., 2016). More recent supervised approaches have utilized deep learning algorithms which use large amounts of training data and computational power to produces predictions without the need for initial feature selection (Barger, Frye, Liu, Dan, \& Bouchard, 2019; Exarchos et al., 2020; Miladinović et al., 2019; Yamabe et al., 2019). Each of these approaches differ from unsupervised learning algorithms which find undetected patterns in the dataset without the need for initial feature identification or 
manual scoring from experts (Sunagawa, Séi, Shimba, Urade, \& Ueda, 2013; Yaghouby, O’Hara, \& Sunderam, 2016).

Despite the excellent performance of the automated behavioral state classifiers highlighted above, many researchers are still dependent upon time-intensive manual classification. Significant functional barriers exist for implementing these automated approaches that typically require extensive computational resources, are constructed within proprietary software, and/or still require significant expert specification of features. To overcome these potential limitations, we developed an artificial neural network (ANN) algorithm for behavioral state classification within the popular, and open-source, R software platform (R Core Team, 2020). This supervised classifier is comprised of four consecutive feed-forward, fully connected layers of artificial neurons. Computational weights of the network are repeatedly adjusted during training in order to help the network 'learn' to classify behavioral state using electrophysiological features of individual 4 s time intervals. This novel ANN: 1 ) is easy to implement, 2) has high classification accuracy, sensitivity, and specificity that are comparable to leading algorithms, 3) has low computational complexity thereby enabling fast algorithm performance with minimal computational resources, and 4) affords flexibility for individual researchers by using freely-available code that is readily customizable in a popular open-source environment. Consequently, this tool serves as a highly accessible and effective automated behavioral state classifier.

\section{Materials and Methods}

\section{Animal Care and Use}

All sample data were obtained from previous experiments conducted at Middlebury College with all methods performed in accordance with the National Institutes of Health Guide for the Care and Use of Laboratory Animals and approved by Middlebury College's Institution Animal Care and Use Committee (approved research protocol \#316-17). Male, Sprague-Dawley rats (3-4 months old, Charles River, Wilmington, $\mathrm{MA}$ ) were obtained and pair-housed upon arrival in clear, plastic rodent caging (16in. $\mathrm{x}$ 7.5in. $x$ 8in.; Teklad TEK-Fresh bedding, Envigo, Indianapolis, IN). Food (Teklad 2020X) and water were provided ad libitum along with in-cage enrichment objects (wooden blocks, pvc tubing, paper towels). After an initial acclimation period (> one week from initial arrival to the vivarium), each rat underwent stereotactic surgery. Here, rats were anesthetized via isoflurane (3.5\% induction, $2-3 \%$ maintenance) and given pre-operative analgesic (Meloxicam; 2mg/kg; MWI, Boise, ID) and antibiotic (Penicillin, 100,000 units $/ \mathrm{kg}$ ) treatments. During surgery, electrodes were implanted for EEG/LFP and nuchal EMG 
97 98

recordings (see sample data set below for additional recording characteristics) and were affixed to the skull using dental acrylic (Lang Dental; Wheeling, IL). A postoperative analgesic (Meloxicam, 2mg/kg) was administered between $12-24 \mathrm{hrs}$ after surgery completion. For the duration of the experiment, rats were then single-housed to minimize potential damage to implanted electrodes and the headstage preamplifier (100x amplification, Pinnacle Technologies, Lawrence, KS). Throughout the experiment, each rat was monitored daily for overt signs of pain/distress including immobility, poor grooming, weight loss, porphyrin staining, postural abnormalities, lack of food/water consumption, and signs of infection around the surgical site. Observation of these signs leads to direct consultations with the attending veterinarian and vivarium staff to determine the appropriate course of action (e.g. treatment or euthanasia). In the present study, no rats were euthanized prior to the conclusion of the experiment. In accordance with the 2013 AVMA Guidelines of Euthanasia, all rats were euthanized via $\mathrm{CO}_{2}$ exposure at the end of the experiment.

\section{Sample Data Set}

Uninterrupted baseline recording days ( $\mathrm{N}=50$ from 11 total rats) were selected from male, SpragueDawley rats (3-4months old). Care was taken to generate a sample of recording days and rats that collectively comprise common recording characteristics across rodent sleep research. Specifically, within each rat, two electrophysiological signals to measure brain activity and one nuchal electromyogram (EMG) were recorded (sampling rate $=250 \mathrm{~Hz} ; 8401$ DACS; Pinnacle Technologies). Rats used in our sample data set had a wide range of recording locations (e.g. prefrontal cortex, motor cortex, parietal cortex) and diverse recording modalities (2 EEGs, 1EEG/1LFP, or 2LFPs). Likewise, these data differed in overall signal quality (e.g. $4.83 \pm 0.56 \%$ mean daily artifact prevalence; range: $0.15 \%$ to 13.48\%). Consequently, this data set represents typical electrophysiological recordings of rodent sleep. Of note, two rats were ultimately excluded from analyses because of very poor EMG signal quality resulting in a final sample data set of 40 baseline days recorded across 9 rats. Details for how EMG signal quality was assessed are presented below as part of our description of our artificial neural network for automated sleep classification.

Trained undergraduate sleep researchers visually scored behavioral state offline in 4s time intervals for each baseline recording day (following conventional sleep research terminology, 4 s time intervals will be referred to as epochs throughout the remainder of the manuscript). Epochs were classified as waking when containing low-voltage, high-frequency EEG/LFP activity and elevated EMG. High-voltage, lowfrequency EEG/LFP activity and an absence of EMG activity was characteristic of NREM sleep, while REM 
128 sleep epochs were classified when low-voltage, high-frequency EEG/LFP activity and an absence of EMG

129 activity were observed.

130

131

132

133

134

135

136

137

138

139

140

141

142

143

144

145

146

147

148

149

150

151

152

153

154

155

156

157

\section{Artificial Neural Network}

To develop an effective research tool with minimal entry barriers for use, we sought to design a freely available, open-source implementation to automatically classify behavioral state using electrophysiological features. With this goal in mind, we chose to implement our algorithm using $R(R$ Core Team, 2020), a freely-available statistical computing platform that has been widely adopted across scientific disciplines (e.g. 58\% of ecological papers published in 2017 used R as their primary analytic tool (Lai, Lortie, Muenchen, Yang, \& Ma, 2019)). Instructions for full implementation of our algorithm and source code can be found at https://github.com/jellen44/AutomaticSleepScoringTool.

Within R, we implemented an artificial neural network (ANN) with four, fully connected sequential layers (256, 128, 32 and 3 nodes) to automatically classify behavioral state in freely behaving rodents. Our ANN was implemented using the Keras package in R with a Tensorflow backend (Abadi et al., 2016). In each of the hidden layers, a weighted sum of inputs is computed, followed by an activation function (rectified linear unit activation $(R e L U)$ ) that introduces nonlinearity and regulates neuronal activation. These weights are adjusted during the training process to improve classification performance. The output layer of this network takes input from the last hidden layer and outputs a vector containing three values, each representing the probability that the epoch is one of the three sleep states. We accomplished this with the SoftMax function, which is commonly used in multiclass classification problems because it takes a K-dimensional vector (the final layer in our network) as an input and uses it to estimate a range of probabilities over a given number of classes (Duan, Keerthi, Chu, Shevade, \& Poo, 2003).

\section{Feature Extraction}

Input for the artificial neural network consisted of 13 EEG-based features per EEG, two EMG features, and two features derived from both EEG and EMG activity. To extract EEG features, we calculated power spectra (Welch's method, Hamming window) for each 4s epoch. From the resultant power spectra, we calculated band-limited power (BLP) within the following frequency bands (delta, 1-4 Hz; theta 4-7 Hz; upper theta 7-9 Hz; alpha 8-12 Hz; beta 13-30 Hz, low gamma $30-50 \mathrm{~Hz}$, medium gamma $50-75 \mathrm{~Hz}$, high gamma $76-125 \mathrm{~Hz}$ ). Five additional features were generated as ratios between frequency bands: 1) beta/delta, 2) beta/low gamma, 3) beta/high gamma, 4) theta/delta, and 5) theta/medium 
158 gamma. EMG features consisted of the root-mean square (RMS) of raw EMG activity and EMG power.

159 As both EMG and gamma activity (Brankack et al., 2010) may serve as useful features for classification of 160 REM sleep, two additional features were generated by summing each EMG feature with medium gamma 161 BLP. Ultimately, all features were z-score normalized prior to input into the ANN model.

\section{ANN Training and Testing}

163 ANN models can operate as supervised learning algorithms that rely upon an initial training set to make predictions about remaining test cases. Our ANN model requires a small initial training set $(560,4 \mathrm{~s}$ epochs; i.e. $2.6 \%$ of daily total) to achieve high classification performance (see Supplemental Figure S1). To increase the likelihood that training features are representative of the diversity of test case activity, we first manually scored 50 pseudorandom series of 10 epochs each. Here random series were selected within the following criteria: 1) they were derived from time points throughout the $24 \mathrm{hr}$ period and 2) periods of NREM sleep, REM sleep, and waking were all included across the 50 series selected). Due to the naturally low prevalence of REM epochs, an additional 60 REM epochs were specifically sought out, manually scored, and incorporated into this training set to ensure sufficient input across all three behavioral states is present for the model. This training set was then algorithmically oversampled

173 (minority classes resampled with replacement) to provide a complete training set with an equal number 174 of epochs across the three behavioral states to be classified in order to avoid highly unbalanced classes due to the low number of REM epochs.

During training, the ANN weights are modified to minimize a categorical cross entropy loss function (a common loss function for discrete classification tasks). To prevent overfitting, we implemented Ridge

178 (L2) regularization $(\beta=0.01)$, which encourages small-weight values by penalizing larger weight values 179 through the loss function (Janocha \& Czarnecki, 2017). Training occurred across 100 complete passes of the training data set (i.e. machine learning epochs) with a batch size of 10 samples. The adaptive moment estimation (Adam) optimizer was used to minimize the loss function with a learning rate of 0.001 to balance accuracy with training time.

Stochastic effects during model training can result in identical inputs producing functional differences in the trained ANN. We therefore implemented an ensemble learning approach (Sagi \& Rokach, 2018), in which outputs from multiple models are combined to improve predictive performance. For each day, the above training procedures were repeated five times and thereby generated five similar, albeit distinct ANNs. Electrophysiological features derived from each 4s epoch not contained within the 
188 training set were used as input features to test the efficacy of the ANNs for classifying behavioral state.

189 Behavioral state was determined as the modal predicted value for each epoch across the five trained 190 ANNs (i.e. most frequent prediction). By averaging prediction certainty across the five ANNs, we also 191 produced a final estimate of the likelihood that a given test epoch was reflective of each behavioral 192 state. For epochs where the mean classification probability across the five ANN models was under $90 \%$, 193 an "uncertain" label was added to that epoch (in addition to the most likely categorical behavioral state output). As detailed more fully in the results section below, this "uncertain" label enables researchers to easily identify a small subset of epochs that 1) are misclassified at a higher rate by the automated ANN and 2) as an option, can be manually rescored to increase classification accuracy while still relying upon automated classification for the vast majority of epochs.

As the above ANNs are trained and tested on an epoch-by-epoch basis (with no between epoch history), these models are unable to directly incorporate sleep/wake history into their behavioral state predictions. To overcome this limitation, once all test epochs have been predicted, we applied a final series of simple heuristic rules to amend the ANN behavioral state predictions to better reflect typical sleep/wake histories. Specifically, we increased REM sleep continuity by rescoring small bouts (<=12s) of scored waking or NREM sleep located between otherwise continuous REM epochs as REM sleep. As direct transitions from waking to REM sleep are exceptionally rare except under pathological conditions (Fujiki, Cheng, Yoshino, \& Nishino, 2009; Mignot et al., 2006), we additionally rescored any epochs of REM sleep that occurred immediately following waking as waking. Lastly, we identified isolated 4s epochs whose state scoring was bounded by epochs that a) differed from the isolated epoch and b) were otherwise scored identically. Once identified, these isolated epochs were rescored to match the immediately preceding/ensuing epochs.

210 Through the course of training and testing these ANNs across our sample data set, it became apparent 211 that the predictive performance of these networks for classifying REM sleep was severely impaired in

212 the absence of a well-functioning EMG. Consequently, prior to training our algorithm first calculates the 213 EMG coefficient of variation (EMG standard deviation/mean RMS EMG). If this standardized quality metric is less than 1.67 (an experimentally derived threshold that appears to identify poor REM sleep classification; see Supplemental Figure S2), a warning to the user that the automated state scoring algorithm is unlikely to successfully score the REM epochs of that particular file is generated. As

217 indicated above, this procedure resulted in the removal of data from two rats in our original sample set. 
219 All analyses of model performance were conducted in Mathworks Matlab (Natick, MA) with additional 220 statistical analyses (correlation, repeated-measures ANOVA, $t$-tests) conducted in SPSS (IBM, Armonk, 221 NY). All data are presented as mean \pm standard error.

222 Performance of the automated sleep scoring algorithm was assessed by comparing its predictions of 223 behavioral state for each epoch with corresponding epochs classified manually. Key metrics calculated 224 include overall accuracy (\% of epochs that were scored identically by automated and manual 225 approaches), state-dependent sensitivity $(T P /(T P+F N))$, and state-dependent specificity (TN/(TN+FP));

226 where TP = True Positive, FN = False Negative, $\mathrm{TN}=$ True Negative, and FP = False Positive. To determine

227 the extent to which training the ANN using data from one baseline day could generalize to a) other

228 baseline days from the same rat and b) other baseline days from different rats, we likewise calculated 229 accuracy, sensitivity, and specificity of the algorithm under these conditions. For within rat 230 generalization, ANNs that were separately trained from each baseline day in the data set were used to 231 predict behavioral state for all other baseline days recorded from that rat. For between rat 232 generalization, ANNs that were separately trained from each baseline day in the data set were used to 233 each predict behavioral state for five baseline days randomly selected from other rats.

234 Lastly, we calculated the effects of using ANN classification on common sleep parameters. Power 235 spectra were calculated for each 4s epoch using Welch's method (Hamming window) and averaged 236 across all epochs of the same behavioral state. To account for potential differences in overall signal 237 strength, spectra were normalized to mean broadband power (i.e. from 0.5-125Hz). To calculate slow 238 wave activity (SWA), we calculated band limited power $(0.5-4 \mathrm{~Hz})$ for each 4 s epoch of NREM sleep and 239 averaged these values across each hour of the light period. Hourly SWA values were then normalized to 240 the mean SWA across the light period.

\section{Results}

\section{Electrophysiological Characteristics Associated with Behavioral State are Useful Features for}

\section{Automated State Classification}

244 Behavioral state scoring, be it manual or algorithmic, relies upon the distinct patterns of neuronal 245 activity characteristic of each behavioral state. Waking epochs are typified by low-voltage, high-

246 frequency EEG activity and variable EMG activity. By contrast, as a function of the large slow waves that 247 predominate NREM sleep, EEG activity during NREM epochs is characteristically high-voltage and low-

248 frequency. REM sleep meanwhile, exhibits EEG activity that is similar to that of waking, but with the 
249

250

251

252

253

254

255

256

257

258

259

260

261

262

263

264

265

266

267

268

269

270

271

272

273

274

275

276

277

278

279

complete absence of EMG activity due to REM-associated motor atonia. While these electrophysiological differences can typically be discerned visually in the time-domain (see Figure 1A for example tracings), quantification of state-dependent activity is usually performed through the extraction of key frequency-domain features. As evident in Figure 1B, EEG band-limited power (BLP) likewise exhibits pronounced state dependency. For example, delta BLP $(0.5-4 \mathrm{~Hz}$; i.e. low-frequency) is clearly elevated during NREM sleep while higher frequency BLP (e.g. theta, gamma) is greater during waking and REM sleep than NREM sleep.

State scoring algorithms consistently rely upon a collection of these electrophysiological features to classify behavioral state (e.g. (Allocca et al., 2019; Gao et al., 2016; Kreuzer et al., 2015; Yan, Qu, Xu, Huang, \& Yao, 2017)). For example, by simply using three features (Delta BLP, Gamma BLP, and EMGRMS), we can reliably segregate the majority of epochs from each behavioral state (Figure 2A). However, as this simple example also shows, many boundary cases remain difficult to classify because a substantial proportion of epochs from each state still overlap within the feature space. To enhance the utility of using electrophysiological features for behavioral state classification, we implemented an artificial neural network (ANN) to predict behavioral state from EEG/EMG features (Figure 2B). During model training, computational weights are continually altered to produce activation maps that accurately predict behavioral state from electrophysiological feature input (see methods and Figure 2C). Once trained, the ANN can uniquely and nonlinearly combine electrophysiological features to generate distinct activation maps characteristic of each behavioral state. Figure 2D depicts activation maps across all four layers of our ANN in response to feature input from manually scored waking, NREM, and REM epochs. With each progressive layer of the ANN, activation maps converge for epochs derived from the same behavioral state and diverge for those derived from differing states. In doing so, the ANN efficiently and effectively uses electrophysiological features to classify behavioral state without researchers needing to know how to precisely combine feature input, a priori.

\section{An Artificial Neural Network Effectively Classifies Behavioral State}

To assess the performance of the ANN detailed above, we compared its behavioral state predictions with manual scoring of the same electrophysiological data by trained undergraduate researchers. Figure 3A/B depict behavioral state hypnograms for a single day using manual and algorithmic scoring, respectively. Here we observe that each scoring method produces a qualitatively similar classification of behavioral state across the $24 \mathrm{hr}$ day. Quantification of the model's performance for this day reveals that the ANN predicted behavioral state with high accuracy, sensitivity, and specificity (Figure 3C).

Peer] reviewing PDF | (2021:05:61856:1:1:NEW 3 Aug 2021) 
280 Across 40 recording days from 9 rats (Figure 3D), the ANN predicted behavioral state with an overall

281 accuracy of $90.87 \pm 0.31 \%$, high sensitivity (Wake: $89.82 \pm 0.50 \%$, NREM sleep: $92.81 \pm 0.58 \%$, and REM

282 sleep: $86.28 \pm 0.99 \%$ ), and high specificity (Wake: $95.18 \pm 0.34 \%$, NREM sleep: $92.71 \pm 0.41 \%$, and REM

283 sleep: $97.18 \pm 0.19 \%)$. Thus, our overall model performance is comparable with that of other high-

284 performing sleep scoring algorithms despite a) the small amount of training data needed (560, 4s

285 epochs; $2.6 \%$ of day's total) and b) in contrast to some algorithms, the ANN predicts behavioral state for

286 all test epochs including those manually-scored as artifact (see Table 1). Moreover, the ANN

287 accomplishes this high performance with rapid computational time (average total run time including fast

288 Fourier transform, feature selection, model training, and prediction output of $223.0 \pm 1$.1s when run on

289 a 2.4GHz Quad-Core Intel Core i5 processor with 8GB 2133 MHz LPDDR3 RAM).

290

291 Although the epochs used to train the ANN models characterized above were randomly selected as

292 described in the methods, the overall performance of these models could nevertheless be dependent

293 upon the specific training epochs that happened to be selected. To address this possibility, we repeated

294 the analyses described above five times for each recording, each time using a different random selection

295 of training epochs. Random differences in training epochs selected produced minimal alterations to

296 model performance; between models trained on different random epochs, low average standard errors

297 were observed for overall accuracy $(0.25 \pm 0.02 \%)$, sensitivity (Wake: $0.55 \pm 0.05 \%$, NREM sleep: $0.53 \pm$

$2980.04 \%$, and REM sleep: $1.81 \pm 0.16 \%$ ), and specificity (Wake: $0.51 \pm 0.05 \%$, NREM sleep: $0.46 \pm 0.04 \%$,

299 and REM sleep: $0.24 \pm 0.02 \%)$. Thus, model performance appears largely unaffected by which specific

$300 \quad 2.6 \%$ of epochs researchers select for model training.

301

302 Although our model compares favorably with other automated scoring approaches, its intended

303 purpose is to serve as a functional tool for sleep researchers. Therefore, more direct assessments of the

304 functional consequences of its use may additionally serve as important metrics of performance.

305 Consequently, across our entire data set, we compared common sleep parameters calculated using our

306 ANN scoring with those calculated using manual scoring. Both scoring methods yield characteristic

307 state-dependent power spectra (Figure 4A); power spectra exhibit clear 1/f power law scaling (Bédard,

308 Kröger, \& Destexhe, 2006), prominent peaks within theta frequencies during waking and REM sleep, and 309 pronounced slow wave activity (SWA) during NREM sleep. Overall, we observed expected significant

310 differences in power spectra as a function of 1 ) behavioral state $\left(F(2,16)=47.26, p=1.9 \times 10^{-7}\right)$ and 2$)$

311 frequency $\left(F(77,616)=72.18, p=2.32 \times 10^{-260}\right)$, but did not observe a significant effect of scoring method 
$312(F(1,8)=0.07, p=0.79)$. Furthermore, we observed a canonical homeostatic decline in SWA across the

313 light period regardless of scoring method employed (Figure 4B; effect of light period time: $F(11,88)=$

$31412.88, p=3.51 \times 10^{-14}$; effect of scoring method: $\left.F(1,8)=3.63, p=0.09\right)$. Thus, key electrophysiological

315 characteristics of behavioral state and homeostatic sleep are not significantly affected by the use of this

316 ANN for behavioral state classification. Lastly, we examined whether ANN classification altered total

317 duration within each behavioral state (Figure 4C) and average episode duration (Figure 4D). During the

318 dark period, the amount of time spent within each behavioral state significantly varied as expected

$319\left(F(2,16)=618.92, p=7.03 \times 10^{-16}\right)$, and this effect was not significantly altered as a function of scoring

320 method $(F(2,16)=1.97, p=0.17)$. In the light period, however, observed significant differences in state

321 duration $\left(F(2,16)=481.88, p=5.06 \times 10^{-15}\right)$ were significantly affected by scoring method $(F(2,16)=$

$32215.83, p=1.6 \times 10^{-4}$ ). Post-hoc $t$-tests reveal that this effect appears largely driven by a significant

323 increase in REM sleep scored by the ANN ( $24.32 \pm 4.60 \%$ more light period REM) at the expense of a

324 significant decrease in light period waking (-9.00 $\pm 1.58 \%)$. Mean episode durations across the entire

325 day, however, were not significantly affected by scoring method (Figure 4D; $F(1,8)=0.42, p=0.54$ ). Thus,

326 the use of this ANN for behavioral state classification does not appear to significantly affect most

327 common sleep parameters, with the exception of an increased prevalence of light-period REM duration

328 that arises at the expense of waking duration.

329

330

\section{Optional Approaches Enhance Scoring Accuracy or Reduce Scoring Time}

Ideally, automated sleep scoring algorithms afford significant time savings with minimal classification

error. To this end, we have shown above that our ANN scoring approach classifies behavioral state with high accuracy, sensitivity, and specificity, while only requiring researchers to manually score $2.6 \%$ of the day. Indeed, increasing the size of the initial training set beyond $2.6 \%$ of the day did not appreciably increase model performance (Figure S1). Certain research applications, however, may necessitate enhanced accuracy even at the expense of additional scoring time. Alternatively, analyses of very large data sets may benefit from reduced scoring time as long as classification performance is not severely diminished. Below we characterize two optional approaches available with our ANN to satisfy these contrasting needs.

In addition to producing a categorical behavioral state prediction for each epoch, our ANN generates an estimate of the certainty of each prediction. To explore how model certainty relates to model performance, we calculated sensitivity and specificity metrics for each behavioral state as a function of 
343 model certainty (Figure 5A). Model sensitivities (Wake: $r(238)=0.80, p=1.10 \times 10^{-54} ; N R E M: r(238)=$ $\left.3440.90, p=8.69 \times 10^{-88} ; \operatorname{REM}: r(238)=0.81, p=4.32 \times 10^{-57}\right)$ and specificities $($ Wake: $r(238)=0.75, p=1.33 \times$ 345 10-44; NREM: $r(238)=0.64, p=4.68 \times 10^{-29}$; REM: $r(238)=0.83, p=2.75 \times 10^{-62}$ ) were all significantly 346 correlated with model certainty. Moreover, these analyses reveal that the vast majority of epochs 347 (88.03 $\pm 1.23 \%)$ were predicted with model certainty greater than $90 \%$. These observations raise the possibility that manually rescoring the relatively small proportion of lower certainty epochs could greatly enhance classification performance. As evident in Figure 5B, manually rescoring epochs associated with lower prediction certainty provides a focused approach that 1) increases sensitivity and specificity across each behavioral state and 2) still affords a significant reduction in researcher time as the ANN automated scores are used for the vast majority of epochs (see Table 1).

Lastly, although the base implementation of our algorithm only requires researchers to manually score $2.6 \%$ of the original file, these small manual scoring requirements may nevertheless present significant time constraints when analyzing very large datasets. Since electrophysiological features associated with each behavioral state are likely to share similar characteristics across recording days and subjects, our trained ANN may be generalizable. To test this possibility, we first trained the ANN on one recording day and then used the trained model to predict behavioral state across all other recording days from the same rat (Figure 6A). Under these conditions, the ANN model maintained high levels of accuracy, sensitivity, and specificity, albeit with a small drop in performance relative to when tested on the same day as trained. Small, but statistically significant (paired $t$-tests), reductions in overall model accuracy ($1.74 \pm 0.41 \%)$, NREM sensitivity $(-1.95 \pm 0.62 \%)$, and Waking/NREM specificity $(-2.38 \pm 0.77 \%$ and $-1.24 \pm$ $0.57 \%$, respectively) were observed. Waking sensitivity and REM specificity were not significantly affected, while a more pronounced and statistically significant decrease in REM sensitivity was present ($6.42 \pm 2.17 \%)$. Collectively, these results appear to indicate that the ANN can effectively classify multiple recording days from the same subject when trained using epochs from a single day. We then sought to determine whether an ANN trained on data from one rat could generalize to recordings from other rats (Figure 6B). While the ANN still maintains moderate performance under these conditions for most parameters of interest (e.g. overall accuracy: $85.33 \pm 0.40 \%$ ), significant decreases in performance were observed relative to ANN models trained on the same day as tested in all performance metrics except REM specificity. REM sensitivity was particularly affected with this approach and significantly

372 decreased by $-27.50 \pm 2.52 \%$. Thus, the ANN appears limited when generalizing to recordings from 373 different rats. 


\section{Discussion}

375 We have developed an artificial neural network that uses EEG/LFP and EMG features to classify

376 behavioral state in rats with high accuracy, sensitivity, and specificity. Quantification of common sleep

377 research parameters (e.g. power spectra, SWA, bout duration, and state durations) is largely unaffected

378 when using this ANN scoring as compared to manual scoring. The ANN requires minimal manual

379 classification, has low computational complexity, and critically is readily implementable using freely

380 available software. Optional features enable researchers to prioritize overall accuracy through manual

381 rescoring of epochs identified as low certainty or further reduce the need for manual scoring by

382 generalizing trained networks across multiple recording days of the same rat. Consequently, this ANN

383 appears to serve as a valuable research tool that increases researcher accessibility to effective and

384 efficient algorithmic classification of behavioral state.

385

386

387

388

389

390

391

392

393

394

395

396

397

398

399

400

401

402

403

Highly accurate behavioral state classification is difficult to achieve despite playing a critical role across a diverse set of research questions. Visual scoring of recorded electrophysiological signals is perhaps the most common approach to solve this challenge yet requires extensive researcher training/time and nevertheless exhibits relatively low inter-rater reliability (83-95\%; (Crisler et al., 2008; Gross et al., 2009; Miladinović et al., 2019; Rytkönen et al., 2011)). Recent advances in machine learning have led to the development of algorithmic classifiers that achieve comparable performance to manual scoring yet afford significant reductions in classification time. Of these, deep learning algorithms have achieved the highest accuracies, yet require extensive computational resources and large training sets (Miladinović et al., 2019; Yamabe et al., 2019). For example, the accuracy of one of these deep learning algorithms is reduced from $96.6 \%$ to $80.5 \%$ when the training set is reduced from 4,200 files to 500 files (Yamabe et al., 2019). These requirements may severely diminish the utility of such approaches for researchers who do not have access to such resources.

By combining supervised learning approaches with deep learning algorithms that utilize simplified neural network architectures, excellent classification performance can be achieved while significantly reducing the need for extensive computational resources as detailed above. Indeed, this approach has previously been successfully implemented for classification of behavioral state in humans (Ronzhina et al., 2012; Schaltenbrand, Lengelle, \& Macher, 1993). Our ANN extends this approach for the classification of behavioral state in rats and achieves high accuracy (90.9\%) despite requiring minimal 
404 manual scoring ( $2.6 \%$ of daily epochs) and low computational resources. During training, ridge

405 regularization and oversampling were undertaken to overcome the imbalanced distribution of 406 behavioral states and increase classification accuracy (Janocha \& Czarnecki, 2017). Z-score 407 normalization of feature input helped alleviate misclassification errors that could arise from 408 distributional shift (Barger et al., 2019). Post-training application of heuristics to transform a subset of 409 the ANN predictions as a function of previous sleep/wake history was used to further improve classification accuracy. Lastly, through automated identification of individual epochs that were predicted with $<90 \%$ certainty by the ANN, our algorithm facilitates optional manual rescoring of a subset of epochs that ultimately increases classification accuracy to 93\%.

413 Although our ANN achieves high overall accuracy, its performance is more limited in terms of REM 414 classification; as compared to manual scoring, the algorithm predicts significantly more REM sleep 415 during the dark period at the expense of waking and only obtains $86.3 \%$ sensitivity for REM epochs. 416 Such performance mirrors that of many different automated approaches as REM classification 417 performance is consistently lower than that for other behavioral states (Exarchos et al., 2020; Rytkönen 418 et al., 2011; Yamabe et al., 2019). Distinguishing between REM sleep and quiet wakefulness presents a 419 significant challenge for automated classifiers because considerable overlap of electrophysiological 420 features is present across these two distinct states. Maximizing feature differences between these 421 states, however, can improve performance. As previously reported (Allocca et al., 2019; Barger et al., 422 2019; Bastianini et al., 2014), we observed that successful classification was highly dependent upon the 423 EMG signal quality. REM sensitivity of our algorithm was correlated with EMG signal quality

424 (Supplemental Figure S2) with REM sensitivity reaching a maximum of $97.8 \%$ for an individual day.

425 Additional features, like heart-rate variability, may further differentiate waking and REM sleep and in 426 doing so improve automated classification (Chouchou \& Desseilles, 2014; Herzig et al., 2018). In the 427 absence of clear feature differentiation, our results indicate that manual rescoring of uncertain epochs 428 represents a practical solution for improving performance, achieving $89.59 \pm 0.66 \%$ average REM 429 sensitivity. Despite some limitations surrounding REM sensitivity, our ANN affords significant benefits as 430 a functional tool for researchers. The quantification of key characteristics inherent to sleep research, 431 including state-dependent power spectra and homeostatic patterns of slow wave activity, are not 432 significantly affected by using this automated scoring approach (Figure 4).

433 Unlike most previous automated behavioral state classifiers (Barger et al., 2019; Crisler et al., 2008; Gao 434 et al., 2016; Gross et al., 2009; Miladinović et al., 2019; Rytkönen et al., 2011; Yan et al., 2017), code for 
435

436

437

438

439

440

441

442

443

444

445

446

447

448

449

450

451

452

\section{3}

454

455

456

457

458

459

460

461

462

our ANN is not only open source but fully written within a commonly used, freely available software environment (R Core Team, 2020). These characteristics enable future researchers to use, and even customize, our initial ANN to meet their specific research needs. Given the similarities in electrophysiological activity and behavioral state classification in rats and mice, we would expect that the current ANN would perform well when classifying behavioral state in both species. Alternatively, the number of neurons in the ANN output layer could be readily altered to classify additional states characteristic of monkey and/or human sleep (Hsieh, Robinson, \& Fuller, 2008; Malafeev et al., 2018), NREM-REM sleep transitions (Benington, Kodali, \& Heller, 1994; Gross et al., 2009), and/or pathological states such as cataplexy (Exarchos et al., 2020). Additionally, feature input could be amended to included novel features like heart-rate variability (Chouchou \& Desseilles, 2014; Herzig et al., 2018) to enhance classification of states of interest. Lastly, even the base architecture of the presented classifier can be modified; although we altered model hyperparameters (e.g. layer number, number of neurons per layer, activation functions, etc.) during model development to enhance classification accuracy, there may be combinations of hyperparameters that we did not test that would yield significant improvement to the model's predictions. Thus, the inherent flexibility of our ANN and ease of access and implementation may enhance the utility of this approach for addressing diverse research questions. Consequently, this ANN represents a valuable tool that can facilitate adoption of highly efficient and accurate automated behavioral state classification.

\section{Conclusions}

Despite recent advances in automated sleep scoring approaches, significant functional barriers still limit their widespread adoption. Here, we present an efficient and accurate automated sleep scoring algorithm that: 1) has minimal computational needs, 2) is freely available, and 3) is coded within a fully open source environment. The use of this behavioral state classifier did not significantly affect most parameters (i.e. state durations, bout lengths, slow wave activity) commonly used in sleep research. Consequently, this accessible and readily implementable ANN may serve as a useful tool for diverse research dependent upon accurate behavioral state classification.

\section{Acknowledgements}

\section{References}


463 Abadi, M., Barham, P., Chen, J., Chen, Z., Davis, A., Dean, J., ... Zheng, X. (2016). TensorFlow: A System 464 for Large-Scale Machine Learning. Proceedings of the 12th USENIX Symposium on Operating 465 Systems Design and Implementation, 265-283.

466

467

468

469

470

471

472

473

474

475

476

477

478

479

480

481

482

483

484

485

Allocca, G., Ma, S., Martelli, D., Cerri, M., Del Vecchio, F., Bastianini, S., ... Gundlach, A. L. (2019).

Validation of 'Somnivore', a Machine Learning Algorithm for Automated Scoring and Analysis of Polysomnography Data. Frontiers in Neuroscience, 13. doi: 10.3389/fnins.2019.00207

Barger, Z., Frye, C. G., Liu, D., Dan, Y., \& Bouchard, K. E. (2019). Robust, automated sleep scoring by a compact neural network with distributional shift correction. PLOS ONE, 14(12), e0224642. doi: 10.1371/journal.pone.0224642

Bastianini, S., Berteotti, C., Gabrielli, A., Del Vecchio, F., Amici, R., Alexandre, C., ... Zoccoli, G. (2014). SCOPRISM: A new algorithm for automatic sleep scoring in mice. Journal of Neuroscience Methods, 235, 277-284. doi: 10.1016/j.jneumeth.2014.07.018

Bédard, C., Kröger, H., \& Destexhe, A. (2006). Does the 1 / f Frequency Scaling of Brain Signals Reflect Self-Organized Critical States? Physical Review Letters, 97(11). doi: 10.1103/PhysRevLett.97.118102

Benington, J. H., Kodali, S. K., \& Heller, H. C. (1994). Scoring Transitions to REM Sleep in Rats Based on the EEG Phenomena of Pre-REM Sleep: An Improved Analysis of Sleep Structure. Sleep, 17(1), 28-36. doi: 10.1093/sleep/17.1.28

Brankack, J., Kukushka, V. I., Vyssotski, A. L., \& Draguhn, A. (2010). EEG gamma frequency and sleepwake scoring in mice: Comparing two types of supervised classifiers. Brain Research, 1322, 5971. doi: 10.1016/j.brainres.2010.01.069

Chouchou, F., \& Desseilles, M. (2014). Heart rate variability: A tool to explore the sleeping brain? Frontiers in Neuroscience, 8. doi: 10.3389/fnins.2014.00402 
486

487

488

Crisler, S., Morrissey, M. J., Anch, A. M., \& Barnett, D. W. (2008). Sleep-stage scoring in the rat using a support vector machine. Journal of Neuroscience Methods, 168(2), 524-534. doi: 10.1016/j.jneumeth.2007.10.027

Duan, K., Keerthi, S. S., Chu, W., Shevade, S. K., \& Poo, A. N. (2003). Multi-category Classification by SoftMax Combination of Binary Classifiers. In T. Windeatt \& F. Roli (Eds.), Multiple Classifier Systems (pp. 125-134). Berlin, Heidelberg: Springer. doi: 10.1007/3-540-44938-8_13

Exarchos, I., Rogers, A. A., Aiani, L. M., Gross, R. E., Clifford, G. D., Pedersen, N. P., \& Willie, J. T. (2020). Supervised and unsupervised machine learning for automated scoring of sleep-wake and cataplexy in a mouse model of narcolepsy. Sleep, 43(zsz272). doi: 10.1093/sleep/zsz272

Fujiki, N., Cheng, T., Yoshino, F., \& Nishino, S. (2009). Specificity of direct transition from wake to REM sleep in orexin/ataxin-3 transgenic narcoleptic mice. Experimental Neurology, 217(1), 46-54. doi: 10.1016/j.expneurol.2009.01.015

Gao, V., Turek, F., \& Vitaterna, M. (2016). Multiple classifier systems for automatic sleep scoring in mice. Journal of Neuroscience Methods, 264, 33-39. doi: 10.1016/j.jneumeth.2016.02.016

Gilmour, T. P., Fang, J., Guan, Z., \& Subramanian, T. (2010). Manual rat sleep classification in principal component space. Neuroscience Letters, 469(1), 97-101. doi: 10.1016/j.neulet.2009.11.052

Gross, B. A., Walsh, C. M., Turakhia, A. A., Booth, V., Mashour, G. A., \& Poe, G. R. (2009). Open-Source Logic-Based Automated Sleep Scoring Software using Electrophysiological Recordings in Rats. Journal of Neuroscience Methods, 184(1), 10-18. doi: 10.1016/j.jneumeth.2009.07.009

Hauglund, N. L., Pavan, C., \& Nedergaard, M. (2020). Cleaning the sleeping brain - the potential restorative function of the glymphatic system. Current Opinion in Physiology, 15, 1-6. doi: 10.1016/j.cophys.2019.10.020 
508 Herzig, D., Eser, P., Omlin, X., Riener, R., Wilhelm, M., \& Achermann, P. (2018). Reproducibility of Heart $509 \quad$ Rate Variability Is Parameter and Sleep Stage Dependent. Frontiers in Physiology, 8. doi:

$510 \quad 10.3389 /$ fphys.2017.01100

511 Hsieh, K.-C., Robinson, E. L., \& Fuller, C. A. (2008). Sleep Architecture in Unrestrained Rhesus Monkeys

512 (Macaca mulatta) Synchronized to 24-Hour Light-Dark Cycles. Sleep, 31(9), 1239-1250.

513 Janocha, K., \& Czarnecki, W. M. (2017). On Loss Functions for Deep Neural Networks in Classification.

514 Schedae Informaticae, 25, 49-59. doi: 10.4467/20838476SI.16.004.6185

515 Kreuzer, M., Polta, S., Gapp, J., Schuler, C., Kochs, E. F., \& Fenzl, T. (2015). Sleep scoring made easy-

$516 \quad$ Semi-automated sleep analysis software and manual rescoring tools for basic sleep research in 517 mice. MethodsX, 2, 232-240. doi: 10.1016/j.mex.2015.04.005

518 Lai, J., Lortie, C. J., Muenchen, R. A., Yang, J., \& Ma, K. (2019). Evaluating the popularity of R in ecology. $519 \quad$ Ecosphere, 10(1), e02567. doi: 10.1002/ecs2.2567

520 Malafeev, A., Laptev, D., Bauer, S., Omlin, X., Wierzbicka, A., Wichniak, A., ... Achermann, P. (2018). Automatic Human Sleep Stage Scoring Using Deep Neural Networks. Frontiers in Neuroscience,

Mignot, E., Lin, L., Finn, L., Lopes, C., Pluff, K., Sundstrom, M. L., \& Young, T. (2006). Correlates of sleeponset REM periods during the Multiple Sleep Latency Test in community adults. Brain, 129(6),

Miladinović, Đ., Muheim, C., Bauer, S., Spinnler, A., Noain, D., Bandarabadi, M., ... Buhmann, J. M. (2019). SPINDLE: End-to-end learning from EEG/EMG to extrapolate animal sleep scoring across experimental settings, labs and species. PLOS Computational Biology, 15(4), e1006968. doi: 10.1371/journal.pcbi.1006968 
530 Nir, Y., Massimini, M., Boly, M., \& Tononi, G. (2013). Sleep and Consciousness. In A. E. Cavanna, A. Nani,

531 H. Blumenfeld, \& S. Laureys (Eds.), Neuroimaging of Consciousness (pp. 133-182). Berlin,

532 Heidelberg: Springer. doi: 10.1007/978-3-642-37580-4_9

Quilichini, P., \& Bernard, C. (2012). Brain state-dependent neuronal computation. Frontiers in

535

536

537

538

539

540

541

542

543

544

545

546

547

548

549

550

551

552

553 Computational Neuroscience, 6. doi: 10.3389/fncom.2012.00077

R Core Team. (2020). R: A Language and Environment for Statistical Computing. Vienna, Austria: R Foundation for Statistical Computing.

Rempe, M. J., Clegern, W. C., \& Wisor, J. P. (2015). An automated sleep-state classification algorithm for quantifying sleep timing and sleep-dependent dynamics of electroencephalographic and cerebral metabolic parameters. Nature and Science of Sleep, 7, 85-99. doi: 10.2147/NSS.S84548

Ronzhina, M., Janoušek, O., Kolářová, J., Nováková, M., Honzík, P., \& Provazník, I. (2012). Sleep scoring using artificial neural networks. Sleep Medicine Reviews, 16(3), 251-263. doi: 10.1016/j.smrv.2011.06.003

Rytkönen, K.-M., Zitting, J., \& Porkka-Heiskanen, T. (2011). Automated sleep scoring in rats and mice using the naive Bayes classifier. Journal of Neuroscience Methods, 202(1), 60-64. doi: 10.1016/j.jneumeth.2011.08.023

Sagi, O., \& Rokach, L. (2018). Ensemble learning: A survey. WIREs Data Mining and Knowledge Discovery, 8(4), e1249. doi: https://doi.org/10.1002/widm.1249

Schaltenbrand, N., Lengelle, R., \& Macher, J.-P. (1993). Neural Network Model: Application to Automatic Analysis of Human Sleep. Computers and Biomedical Research, 26(2), 157-171. doi: 10.1006/cbmr.1993.1010

Stephenson, R., Caron, A. M., Cassel, D. B., \& Kostela, J. C. (2009). Automated analysis of sleep-wake state in rats. Journal of Neuroscience Methods, 184(2), 263-274. doi: 10.1016/j.jneumeth.2009.08.014 
554 Sunagawa, G. A., Séi, H., Shimba, S., Urade, Y., \& Ueda, H. R. (2013). FASTER: An unsupervised fully

$555 \quad$ automated sleep staging method for mice. Genes to Cells, 18(6), 502-518. doi:

$556 \quad 10.1111 /$ gtc.12053

557 Yaghouby, F., O'Hara, B. F., \& Sunderam, S. (2016). Unsupervised Estimation of Mouse Sleep Scores and

558 Dynamics Using a Graphical Model of Electrophysiological Measurements. International Journal

559 of Neural Systems, 26(4), 1650017. doi: 10.1142/S0129065716500179

560 Yamabe, M., Horie, K., Shiokawa, H., Funato, H., Yanagisawa, M., \& Kitagawa, H. (2019). MC-SleepNet:

$561 \quad$ Large-scale Sleep Stage Scoring in Mice by Deep Neural Networks. Scientific Reports, 9(1),

562 15793. doi: $10.1038 / s 41598-019-51269-8$

563 Yan, M.-M., Qu, W.-M., Xu, X.-H., Huang, Z.-L., \& Yao, M.-H. (2017). Entire Frequency Domain Analysis of 564 Rodent EEG and EMG Recordings Using Relative Thresholds. Sleep and Vigilance, 1(1), 13-19.

565 doi: 10.1007/s41782-017-0004-y

566 


\section{Figure 1}

Characteristic state-dependent differences in electrophysiological activity.

A) Representative EEG, EMG, and RMS EMG tracings across 8s periods of wake, NREM sleep, and REM sleep from an individual rat. B) z-scored electrophysiological features depict statedependent variation in EEG and EMG activity. For complete description of EEG/EMG feature input to the neural network classifier, see methods.

A

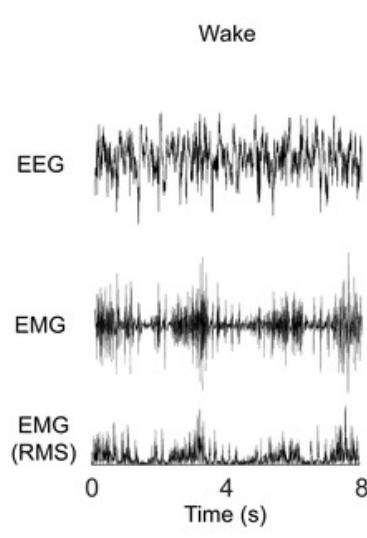

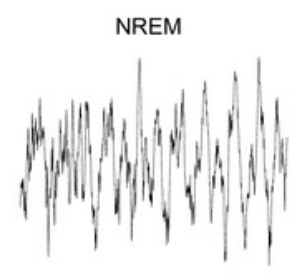
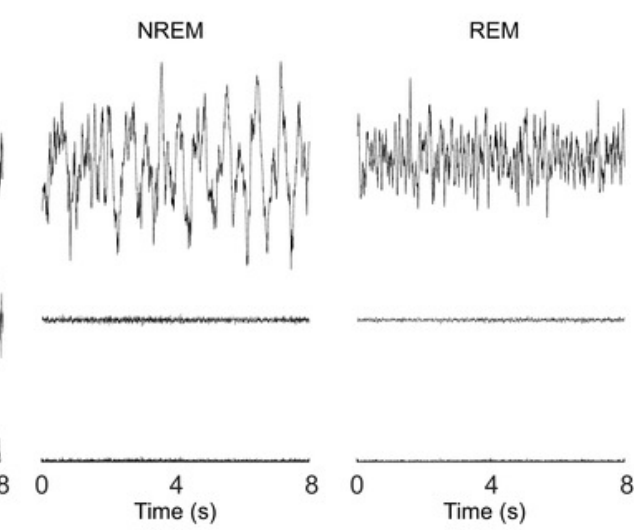

B

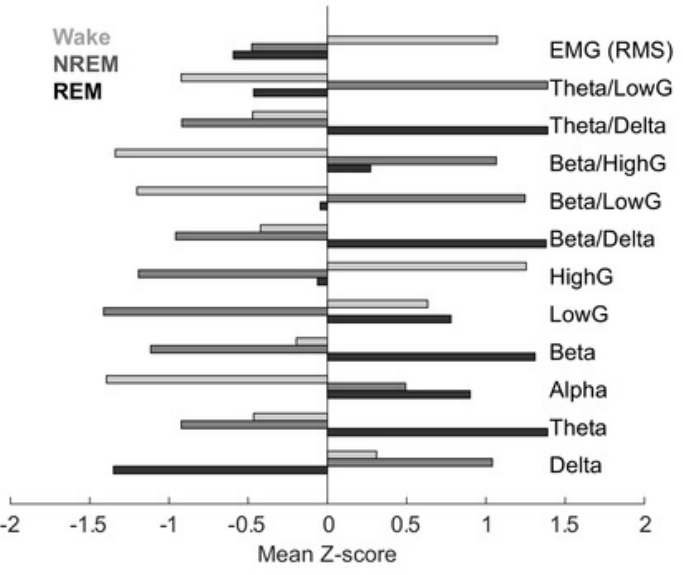




\section{Figure 2}

Artificial neural network reliably predicts behavioral state from EEG and EMG features.

A) Example data from an individual rat depicts how electrophysiological features alone reliably segregate behavioral state for most $4 \mathrm{~s}$ epochs, but fail to resolve ambiguity of many boundary cases. B) Artificial neural network architecture employed in the current study to enhance the predictive utility of EEG/EMG features for behavioral state classification (see methods for detailed characteristics of input features used). C) Reductions in categorical cross-entropy loss and increased prediction accuracy of training data occur across model training. Data depict training of a single model. D) Activation maps across the four layers of our artificial neural network in response to EEG/EMG features. Within each layer, artificial neurons are depicted as colored boxes with color representing the amount of activation of that neuron in response to input features from a $4 \mathrm{~s}$ epoch. Each of the six rows depict activation maps from a unique $4 \mathrm{~s}$ epoch from the same rat; two epochs of each behavioral state were chosen to show similarities and differences in activation within and across data from each behavioral state. Note, activation of specific neurons in the output layer corresponds directly with the predicted output of the model which matches the manual scoring for each of the epochs presented above. 
A

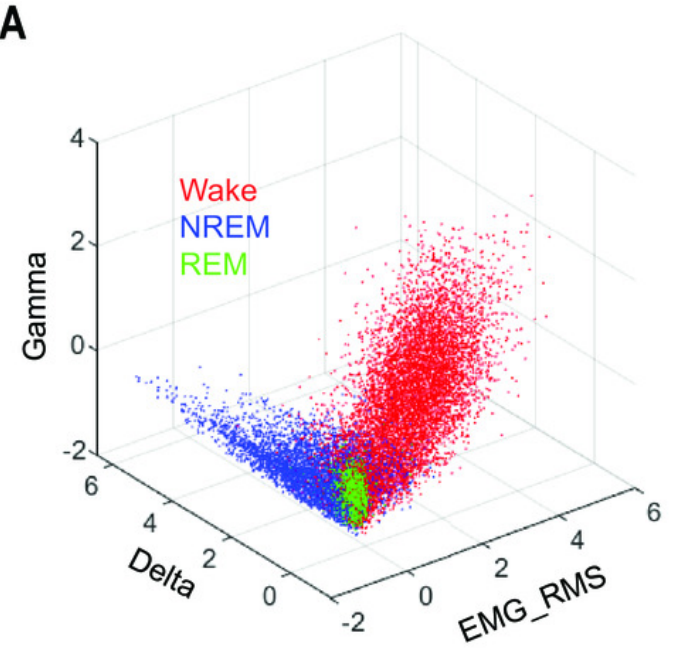

B

C

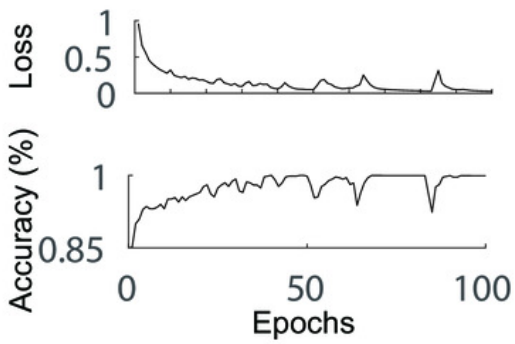

D

Layer 1

(Hidden; 256)
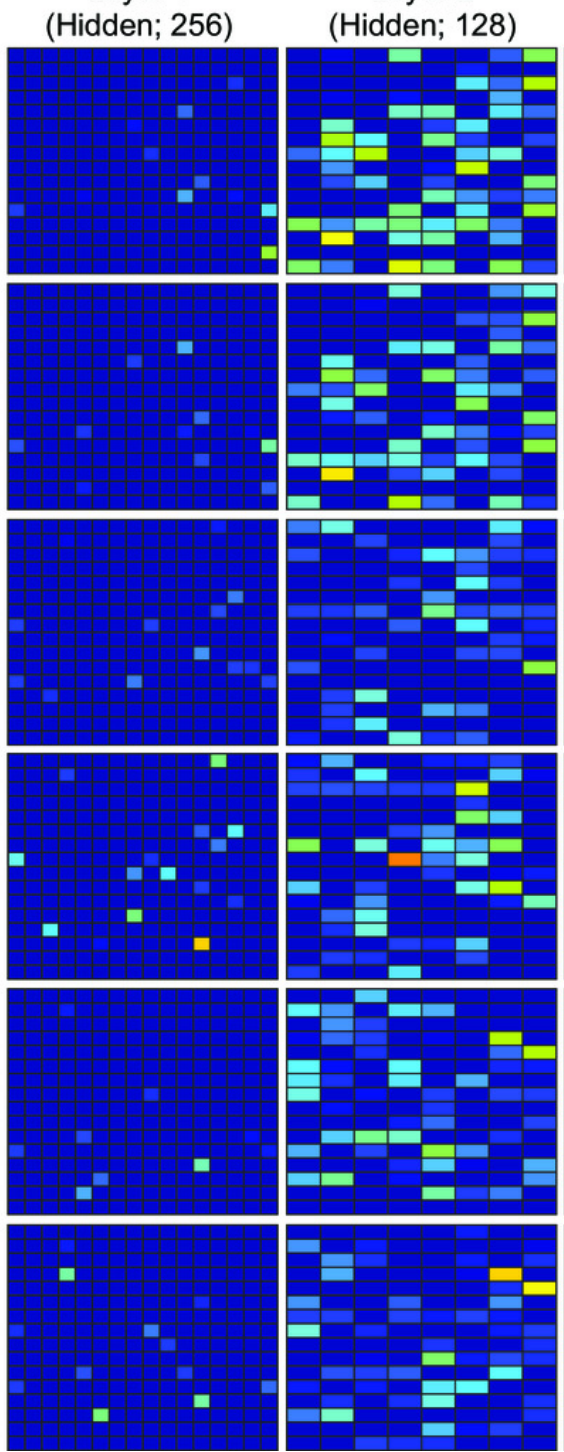

Layer 3 (Hidden; 32)
Layer 4

(Output; 3)

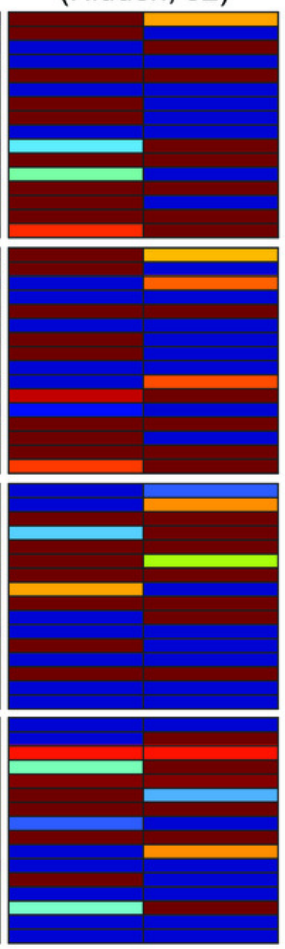

W NR R

W NR R

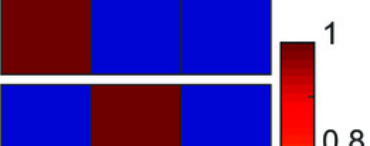

W NR $R$

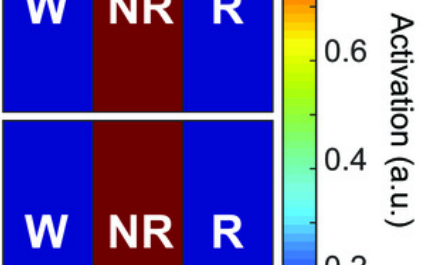

W NR R

W NR R 


\section{Figure 3}

Sleep scoring performance of artificial neural network.

A/B) Example hypnograms from an individual rat depict behavioral state as characterized by manual or algorithmic scoring, respectively. Bottom bar depicts the 12/12hr daily light cycle.

C) Quantification of algorithm accuracy, sensitivity, and specificity for example data

presented in A. D) Accuracy, sensitivity, and specificity of algorithmic scoring across all days ( $\mathrm{N}=40$ total from 9 rats). Acc - Overall model accuracy, W - Waking, NR - NREM sleep, R REM sleep.

A

A

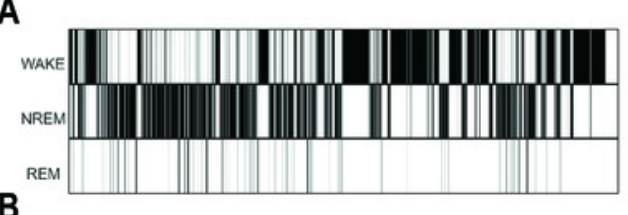

B

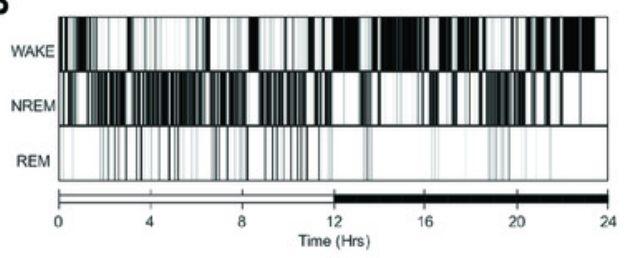

C

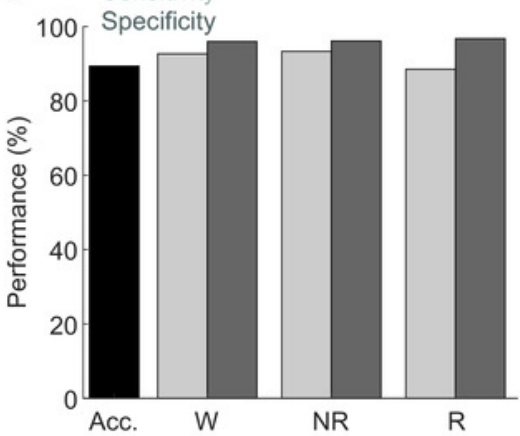

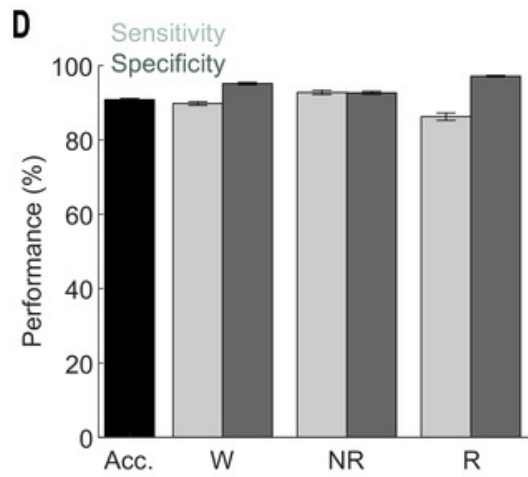




\section{Figure 4}

Quantification of common sleep parameters is highly similar when using manual or algorithmic scoring.

A) No significant differences in state-dependent power spectra were observed when computed using manual/algorithmic scoring methods. Each line depicts mean $+/$ - standard error. B) Slow wave activity (SWA) exhibits a similar homeostatic decline across the light period with both scoring methods. C/D) Total durations and mean bout durations are similar across both scoring methods, with the exception of a significant increase in light-period REM duration and decrease in light-period waking duration. $* p<0.05 \mathrm{~W}-$ Waking, NR - NREM sleep, R - REM sleep.
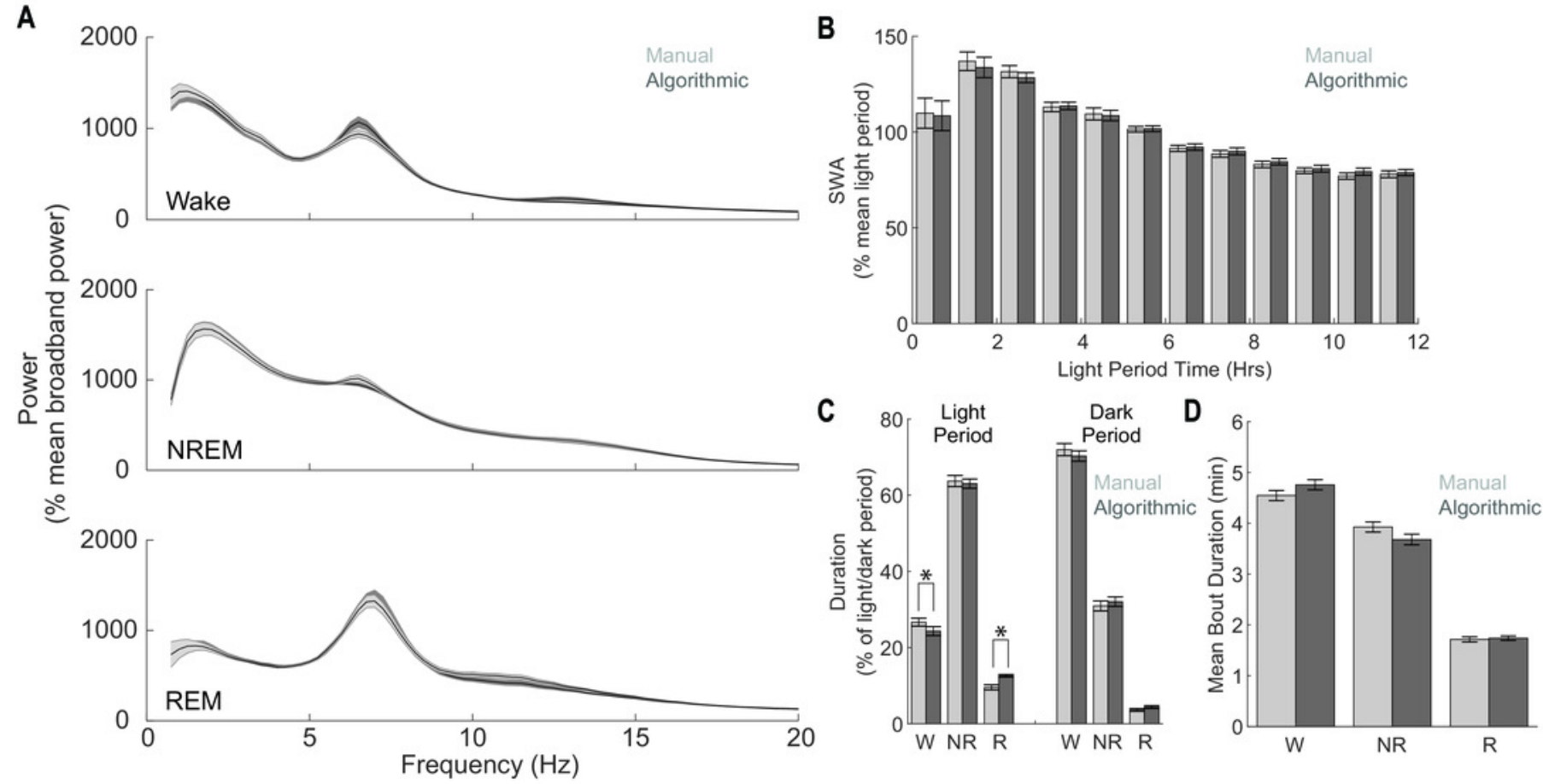


\section{Figure 5}

Algorithm performance corresponds with individual epoch prediction probabilities.

A) The algorithm has high confidence ( $>90 \%$ ) for the majority of the $4 \mathrm{~s}$ epochs scored across

$24 \mathrm{hrs}$ and therein produces predictions with high sensitivity and specificity. B)

Sensitivity/Specificity across the entire day are enhanced through manual rescoring of the subset of epochs that have lower $(<90 \%)$ prediction confidence. Insets depict overall model performance after manual rescoring all epochs with lower prediction confidence. Note sensitivity/specificity in A) reflect only those epochs within the model certainty bin while in B) reflect performance across all epochs. Within each bin, data triplets reflect wake, NREM sleep, and REM sleep respectively.
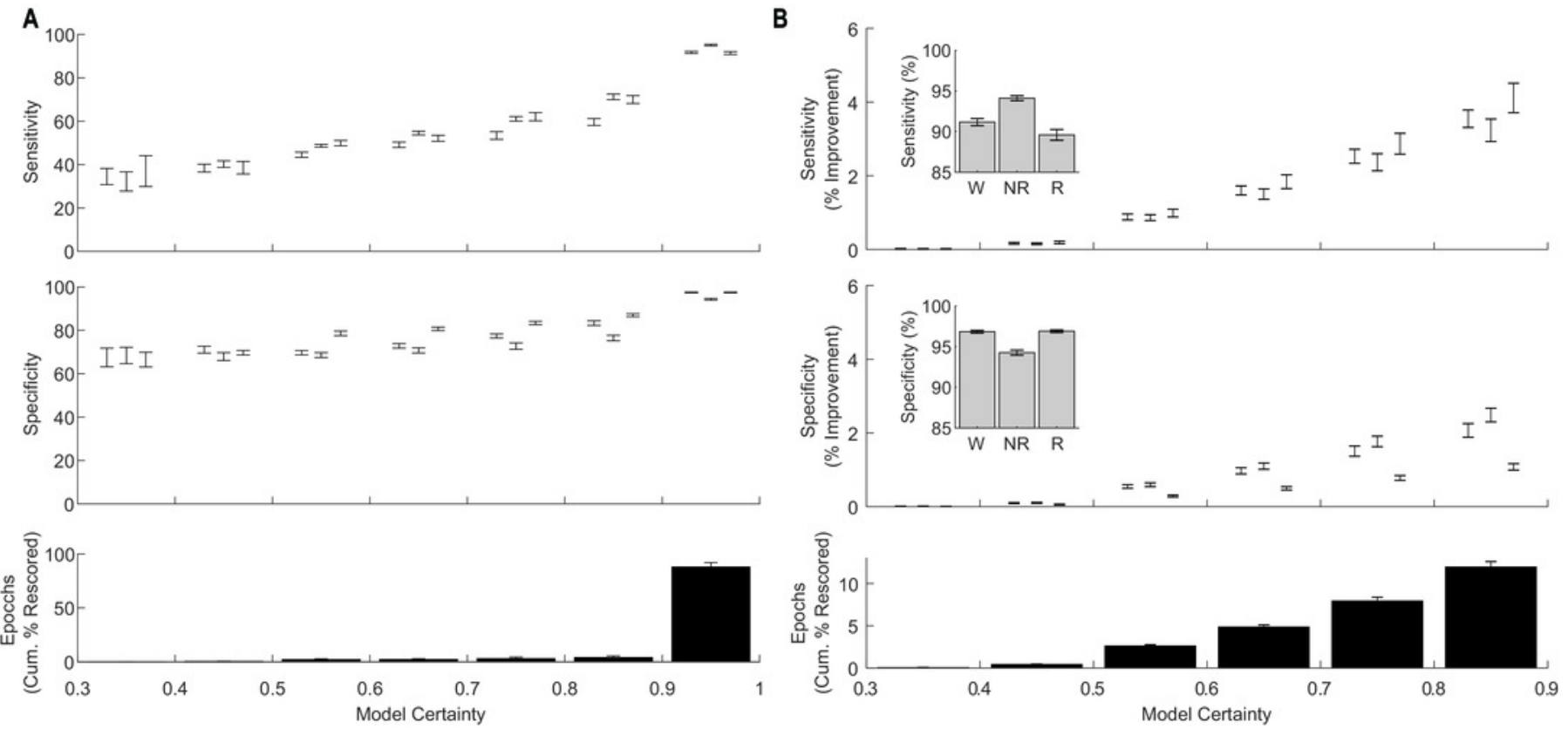


\section{Figure 6}

Classification performance of the trained algorithm when tested across different recording days and rats.

A/B) State-dependent classification accuracy, sensitivity, and specificity when testing the algorithm on different recording days from the same rat or different rats, respectively. Dark asterisk bars depict mean model performance when tested on the same day as trained (i.e. Figure 3D). Acc - Overall model accuracy, W - Waking, NR - NREM sleep, R - REM sleep.
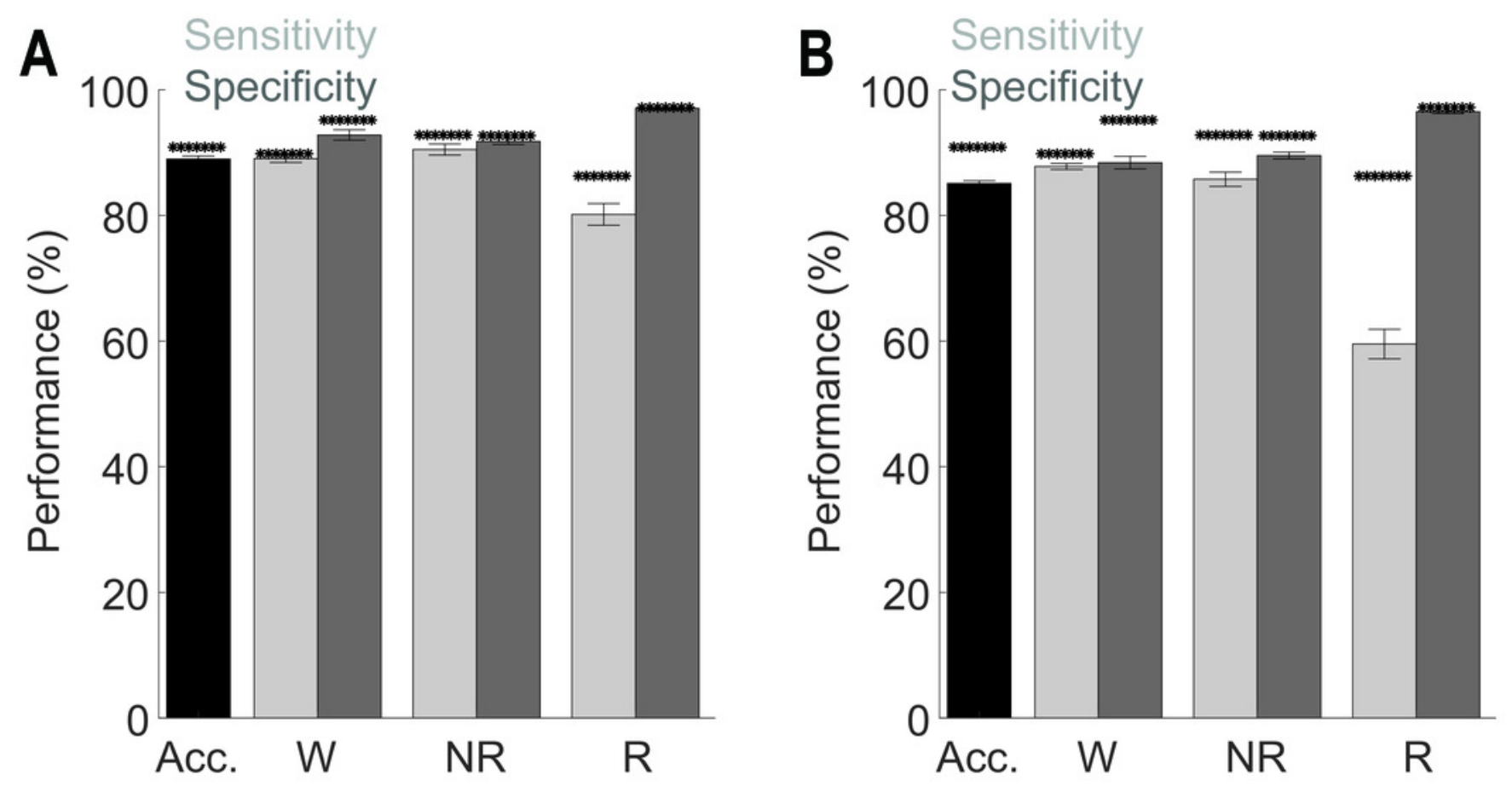


\section{Table $\mathbf{1}$ (on next page)}

ANN model performance as compared to previous automated sleep scoring approaches to classify rodent behavioral state. 
Manual Scoring (\% of total epochs)
Freely-Available, Open Source (Environment)

$\begin{array}{lccc}91 & 2.6 & \text { Yes (R) } & \text { Yes } \\ 92 & 2.6 & \text { Yes (R) } & \text { No } \\ 93 & \sim 14 & \text { Yes (R) } & \text { Yes } \\ 89 & & & \text { Yes } \\ & 2.6 \text { (training day) } & \text { Yes (R) } & \end{array}$

\section{Current Manuscript \\ Current Manuscript \\ Current Manuscript \\ Current Manuscript}

\section{Artificial Neural Network}

Artificial Neural Network

Artificial Neural Network

+ Manual Rescore

Artificial Neural Network

+ Same Rat Generalization

\begin{tabular}{|c|c|c|}
\hline Exarchos, et al., 2020 & Convolution Neural Network & 93 \\
\hline Exarchos, et al., 2020 & Dimension Reduction + Clustering & 89 \\
\hline Yamabe et al., 2019 & $\begin{array}{l}\text { Convolution Neural Network } \\
\text { + Long Short-Term Memory }\end{array}$ & 97 \\
\hline Miladinovic et al., 2019 & $\begin{array}{l}\text { Convolution Neural Network } \\
\text { + Hidden Markov Model }\end{array}$ & $93-9$ \\
\hline Miladinovic et al., 2019 & $\begin{array}{l}\text { Convolution Neural Network } \\
\text { + Hidden Markov Model }\end{array}$ & 89 \\
\hline Barger et al., 2019 & $\begin{array}{l}\text { Convolution Neural Network } \\
+ \text { Mixture z-scoring }\end{array}$ & 97 \\
\hline Allocca et al., 2019 & Support Vector Machine & 0.94 \\
\hline Yan et al., 2017 & Threshold Decision Tree & 91 \\
\hline Gao et al., 2016 & Multiple Classifier System & \\
\hline Kreuzer et al., 2015 & Threshold Decision Tree & 91 \\
\hline Bastianini et al., 2014 & Threshold Decision Tree & $89-9$ \\
\hline Yaghouby et al., 2013 & Hidden Markov Model & 90 \\
\hline Rytkonen et al., 2011 & Naïve Bayes Classifier & 93 \\
\hline Gross et al., 2010 & Threshold Decision Tree & 80 \\
\hline Stephenson et al., 2009 & Threshold Decision Tree & 89 \\
\hline Crisler et al., 2008 & Support Vector Machine & 96 \\
\hline
\end{tabular}

1

\begin{tabular}{|c|c|c|c|}
\hline 93 & $\sim 15$ & Yes (Google Colab) & Yes \\
\hline 89 & 0 ; unsupervised & Yes (Google Colab) & Yes \\
\hline 97 & Not reported & Yes (Python) & Yes \\
\hline 93-99 & $\sim 9$ & Yes (Torch) & No \\
\hline 89 & $\sim 9$ & Yes (Torch) & Yes \\
\hline 97 & $\sim 1-2$ & Partially ${ }^{2}$ (Matlab - GUI) & Yes \\
\hline $0.94^{1}$ & $<1$ & Partially² (Matlab - GUI) & No \\
\hline 91 & 0; Threshold & No (Matlab) & Yes \\
\hline$\sim 95$ & $\sim 9$ & No (Matlab) & No \\
\hline 91 & 0; Threshold & Partially² (LabVIEW - GUI) & No \\
\hline $89-97$ & 0; Threshold & No (Matlab) & Yes \\
\hline 90 & 0 ; unsupervised & No (Matlab) & $\sim 5 \%$ of epochs excluded \\
\hline 93 & 5 & No (Matlab) & Only days with $<5 \%$ artifact \\
\hline 80 & 0; Threshold & Partially² (Matlab - GUI) & Yes \\
\hline 89 & 0; Threshold & Yes (Spreadsheet) & No \\
\hline 96 & $\sim 4$ & No (Matlab) & No visually-scored artifacts \\
\hline
\end{tabular}

${ }^{2}$ Standalone open-source tools enable algorithm implementation, but not editing 